\title{
WIND TURBINE MULTIVARIABLE OPTIMAL CONTROL BASED ON INCREMENTAL STATE MODEL
}

\author{
José Miguel Adánez Basil Mohammed Al-Hadithi $\quad$ Agustín Jiménez
}

\begin{abstract}
The multivariable optimal control of a wind turbine by an approach based on incremental state model is proposed. The advantages of incremental state model in comparison with the non incremental one are that the control action cancels steady state errors and incremental state solves the problem of computing the target state, choosing zero as an objective. Linear Quadratic Regulator (LQR) and optimal state observer are applied. The effectiveness of the proposed control method, over the non incremental one, is examined by applying the linear controllers to the nonlinear wind turbine model. The results show that incremental LQR control presents good transient response and zero steady state errors, even in presence of disturbances, nonlinearities and modelling errors.
\end{abstract}

\section{INTRODUCTION}

Wind turbine technology has undergone rapid development in response to increased demand for renewable energy. At the beginning of 2017, installed capacity worldwide was about $485 \mathrm{GW}$, see Fig. 1, installing more than $50 \mathrm{GW}$ in 2016 [1]. Technological advances in wind turbines make necessary the design of effective control systems, whose main objective is to get the maximum possible energy and to increase the wind turbine lifetime by minimizing loads at the structure [2].

There are several control loops responsible of optimum operation of the wind turbine [3,4]: rotor angular speed control; torque control [5]; pitch control (angle of blades) [6]; yaw control (orientation of the wind turbine) [7]; and power control loops [8]. Currently, the problem of wind turbine control in industry is solved by implementing each control loop separately using conventional proportional integral (PI) or proportional integral derivative (PID) controllers [9], and minimizing the couplings among loops by iterative adjustments. However, the new wind turbine structures, larger, more flexible and operating in uncertain environments, make traditional controllers designs insufficient. Therefore the need for advanced control methods are increased $[10,11]$.

Although the wind turbine industry mainly uses classical controllers, some researchers have also tried some modern control techniques. For example, a Quantitative Feedback Theory (QFT) controller has been applied in an industrial turbine [12]. Also, multivariable state space $\mathrm{H}_{\infty}$ control has been tested in the turbine CART 3 (Controls Advanced Research Turbine 3 -bladed) with very promising results [13,14].

The speed control of induction motor fed by wind turbine using imperialist competitive algorithm is proposed in [15]. The proposed design problem of speed controller is established as an optimization problem. Imperialist competitive algorithm is adopted to search for optimal controller parameters by minimizing the time domain objective function.

In [16], the authors propose Ant Lion optimization algorithm for optimal allocation and sizing of renewable distributed generation sources in various distribution networks. Allocation and sizing of distributed generation have greatly affected on the system losses. Photovoltaic systems and wind turbines are considered here as sources of distributed generation. The most appropriate candidate buses for installing distributed generation are suggested using loss sensitivity factors and the algorithm is employed to deduce the locations of distributed generation and their sizing from the elected buses.

A theoretical framework for adaptive control of a wind energy conversion system, involving a squirrel cage induction generator, is presented in [17]. A multi-loop 


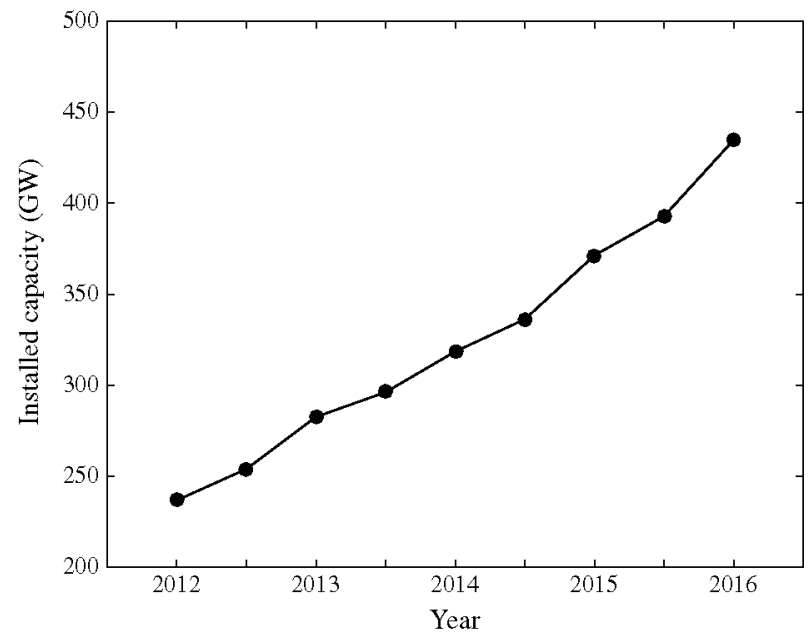

Fig. 1. Total installed capacity.

nonlinear controller is designed to meet two main control objectives, the speed reference optimization in order to extract a maximum wind energy whatever the wind speed, and power factor correction to avoid net harmonic pollution. These objectives are achieved despite the mechanical parameters uncertainty.

A cascaded multivariable nonlinear control is performed in [18]. The inner loop controls the generator torque and stator flux, while the outer loop controls the rotor angular speed.

In [19], a classical multivariable control is developed by decoupling and feedforward compensation. The decoupling is incorporated to minimize interaction effects among classical PI control loops. Feedforward compensator is included to reduce the effects of wind disturbances and electrical load changes.

Multivariable coupling effects are studied for load reduction in wind turbines [20]. They prove that multiple single-input single-output (MSISO) controller is easy to understand but it creates couplings among control loops, and it has to be improved by iterative adjustment. On the other hand, multi-input multi-output (MIMO) controller in state space form can achieve higher load reductions without any additional filtering.

A gain scheduling method based on output interpolation of some local controllers independently designed is presented for wind turbine control [21]. The local controllers are designed using $\mathrm{H}_{\infty}$ optimal control method for the multivariable state space model defined at each local point.

An optimal control system based on feedforward and state-feedback controller is designed for a fuel processing system [22], using a loop transfer recovery method and a generalized linear quadratic Gaussian method. A new methodology is proposed to design digital PID controllers for multivariable systems with time delays [23]. Most of the parameters are systematically tuned using state-feedback and state-feedforward LQR approach. An extension for multiple time delays is presented in [24].

In [25], the authors investigates the problem of finite-time optimal tracking control for dynamic systems on Lie groups for the situation when the tracking time and the cost functions need to be considered. A control law is designed to track a desired reference trajectory at the given time and to guarantee the cost functions to be optimal.

A design strategy of robust disturbance observer is proposed systematically for stable non-minimum phase systems in [26]. This strategy synthesizes the internal and robust stability, relative order and mixed sensitivity design requirements together to establish the optimization function. The optimal solution is obtained by standard $\mathbf{H}_{\infty}$ control theory under the condition of guarantying the presented requirements.

In [27], a predictive control algorithm called Difference Equation Matrix Model (DEMM) was developed and applied to the control of a wind turbine. This algorithm is considered a mixture of Dynamic Matrix Control (DMC) [28] and Recursive Generalized Predictive Control (RGPC) [29]. Several advantages over the two methods are obtained. In comparison with [28], DEMM control decreases the computational cost, which make it compatible for real time applications. Also, comparing with [29], DEMM control presents zero steady state error, good and robust performance in front of disturbances and model uncertainties.

The incremental state model is presented in [30] to model multivariable nonlinear delayed systems expressed by a generalized version of the Takagi-Sugeno (T-S) fuzzy model. The advantages of the incremental state model compared with the non-incremental one have been defined. First, it solves the problem of computing the target state, choosing zero incremental state as an objective. Second, the control action in an incremental form is equivalent to introduce an integral action. Third, incremental state model makes the affine terms disappear.

In [30] a new optimal state observer is also presented. In this way, and combined with the LQR method $[31,32]$, a local optimization is produced at each operating point defined by the T-S fuzzy model. A multivariable thermal mixing tank system is chosen to evaluate the effectiveness of the proposed methods.

This paper is organized as follows: In Section II, the wind turbine dynamic model is described. In Section III, we detail the modelling and control methodology for both incremental and non incremental state models. 
Results of proposed control algorithms applied to the wind turbine model are shown and analyzed in Section IV. Finally, the conclusions are presented in Section V. The nomenclature is added in the appendix in Table A1 and the list of abbreviations is added in Table A2.

\section{WIND TURBINE MODEL}

In this section, the wind turbine dynamic model is described. The block diagram, see Fig.2, represents the multivariable behaviour of the wind turbine. The system manipulated inputs are blades angle reference $\beta_{r}$ and field current reference $I_{f_{s}}$. The non-manipulated inputs

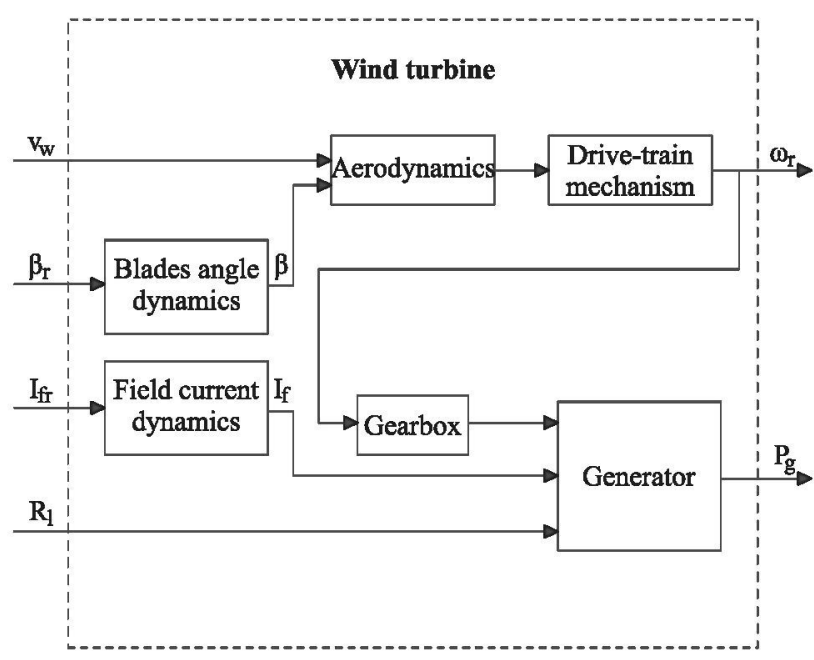

Fig. 2. Wind turbine diagram.
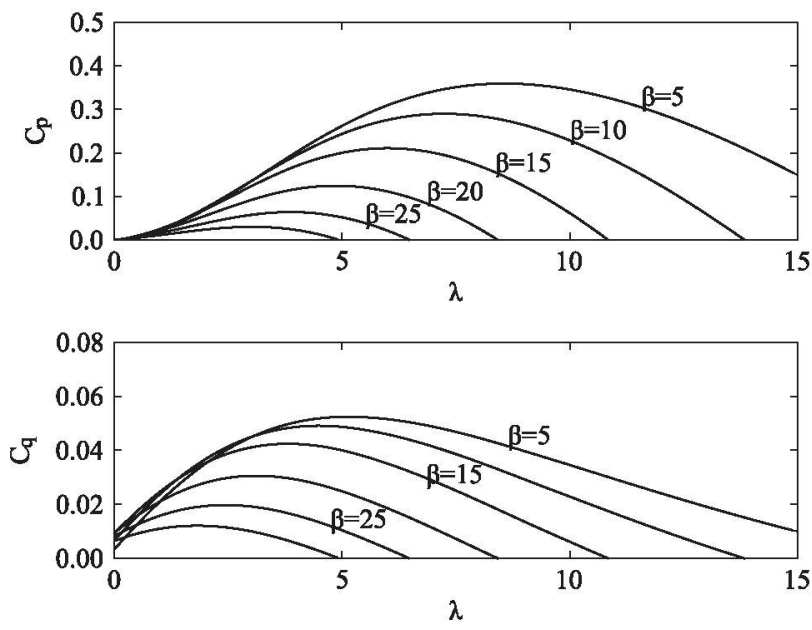

Fig. 3. Power $C_{p}$ and mechanical $C_{q}$ coefficients. are wind speed $v_{w}$ and electrical line resistance $R_{L}$. The system outputs are rotor angular speed $\omega_{r}$ and generated power $P_{g}$.

The generator configuration used for the wind turbine model is a Wound Rotor Syncrhonous Generator (WRSG), in which the electromagnetic torque can be controlled by a current applied to the rotor (field current reference $I_{f_{s}}$ ). This allows simplifying the power converter that is connected to the generator, thus it can be considered a simple rectifier diode bridge. It is considered that the wind turbine is equipped with a mechanism which allows varying the blades angle using a pressure oil valves group commanded by a blades angle reference signal $\beta_{r}$. The wind turbine model is supposed to have some ideal sensors, which allows the measurement of the most important variables [3]: inductive linear positioning sensors could be used for measuring the blades angle $\beta$, ammeters could be used for measuring the field current $I_{f}$, anemometers could be used for measuring the wind speed $v_{w}$, incremental encoders could be used for measuring the rotor angular speed $\omega_{r}$ and power measurement devices could be used for measuring the generated power $P_{g}$.

The nonlinear model equations of the wind turbine, obtained from different literature sources, for example [19], are summarized in Table I. We have used as power and mechanical coefficient [33], which model the blades aerodynamic behaviour, those shown in Fig. 3. Wind turbine model includes the transfer functions representing the blades angle $\beta$ and field current $I_{f}$ dynamics, with respect to their reference signals:

$$
\beta(s)=\frac{1}{\left(1+T_{\beta} s\right)^{2}} \beta_{r}(s)
$$

Table I. Nonlinear model equations summary.

\begin{tabular}{lc}
\hline Variable & Equation \\
\hline Aerodynamics & $\lambda=\frac{R \omega_{r}}{v_{w}}$ \\
Ratio $\lambda$ & $C_{p}=\lambda C_{q}(\lambda, \beta)$ \\
Power coefficient & $\tau_{m}=0.5 \rho \pi R^{3} v_{w}^{2} C_{q}$ \\
Mechanical torque & \\
Drive-train mechanism & $J_{t} \dot{\omega}_{r}=\tau_{m}-B_{t} \omega_{r}-\tau_{g}$ \\
Low speed shaft dynamics & $\omega_{g}=N \omega_{r}$ \\
High speed shaft angle rate & $\tau_{g}=\frac{N}{\eta_{g} \eta_{m}} \tau_{e m}$ \\
Electromagnetic torque & $P_{g}=\tau_{e m} \omega_{g}$ \\
Electrical generator & $\tau_{e m}=K^{2} \frac{R_{L}}{R_{L}^{2}+X_{g}^{2}} I_{f}^{2} \omega_{g}$ \\
Generated power & $X_{g}=\alpha N \omega_{r}$ \\
Electromechanical torque &
\end{tabular}


Table II. Wind turbine parameters.

\begin{tabular}{lcc}
\hline Parameter & Symbol & Value \\
\hline Rotor radius & $R$ & $4(\mathrm{~m})$ \\
Wind density & $\rho$ & $1.225\left(\mathrm{~kg} / \mathrm{m}^{3}\right)$ \\
Gear ratio & $N$ & 11 \\
Inertia moment & $J_{t}$ & $15\left(\mathrm{~m}^{2} \mathrm{~kg}\right)$ \\
Viscous friction & $B_{t}$ & $0.2(\mathrm{Nms} / \mathrm{rad})$ \\
Generator constant & $K$ & $0.223(\Omega \mathrm{s} / \mathrm{rad})$ \\
Inductance & $\alpha$ & $0.0178(\Omega \mathrm{s} / \mathrm{rad})$ \\
Generator efficiency & $\eta_{g}$ & 0.6 \\
Mechanism efficiency & $\eta_{m}$ & 0.4 \\
Blades angle time constant & $T_{\beta}$ & 1 \\
Field current time constant & $T_{f}$ & 0.5 \\
\hline
\end{tabular}

Table III. Wind turbine operating point.

\begin{tabular}{lc}
\hline Variable & Equation \\
\hline Wind speed $v_{w}$ & $10.55(\mathrm{~m} / \mathrm{s})$ \\
Electrical line resistance $R_{L}$ & $4(\Omega)$ \\
Field current $I_{f}$ & $2(\mathrm{~A})$ \\
Blades angle $\beta$ & $7\left(^{\circ}\right)$ \\
Rotor angular speed $\omega_{r}$ & $32.24(\mathrm{rad} / \mathrm{s})$ \\
Generated power $P_{g}$ & $1791.8(\mathrm{~W})$ \\
\hline
\end{tabular}

$$
I_{f}(s)=\frac{1}{1+T_{f} s} I_{f_{r}}(s)
$$

The order of the wind turbine subsystems dynamics is taken into account as follows: the first order drive-train mechanism dynamic, the second order blades angle dynamic and the first order field current dynamics. Thus, the overall system dynamics for both outputs, rotor angular speed $\omega_{r}$ and generated power $P_{g}$, is fourth order.

Table II summarizes the wind turbine parameters and Table III describes one of the possible operating points proposed for the wind turbine nominal operation, in which we have linearized the nonlinear wind turbine model to obtain the proposed linear controllers.

We have modelled the hardware requirements of the actuators in equations 1 and 2, and the sensors are supposed to be ideal, so they have not been modelled. We have considered the wind speed $v_{w}$ as non-manipulated measurable input (measurable disturbance), but we have not modelled it since the results are developed in front of step disturbances in the wind speed, in order to clearly show the proposed controllers behaviour.

\section{MULTIVARIABLE MODELLING AND CONTROL METHODOLOGY}

Discrete model based on input-output identification allows, through a systematic method, obtaining discrete and incremental state models of any linear system, or at least linearized ones. Once the state model is obtained, we can apply the appropriate controller and observer to achieve the control objectives.

\subsection{Multivariable discrete state model}

Given a multivariable discrete system with $p$ manipulated inputs $u \in \Re^{p}, r$ non-manipulated measurable inputs (measurable disturbances) $d \in \mathfrak{R}^{r}$ and $q$ outputs $y \in \mathfrak{R}^{q}$, where $q \leq p$, its model in difference equations can be obtained by input-output identification. Affine terms can be used to avoid using incremental values around operating point:

$$
\begin{aligned}
y_{i}(k+1)= & a_{i 0}+a_{i 1} y_{i}(k)+a_{i 2} y_{i}(k-1)+\cdots \\
& +a_{i n_{i}} y_{i}\left(k-n_{i}+1\right)+\sum_{j=1}^{p}\left(b_{i j 1} u_{j}(k)+\right. \\
& \left.+b_{i j 2} u_{j}(k-1)+\cdots+b_{i j n_{i}} u_{j}\left(k-n_{i}+1\right)\right) \\
& +\sum_{l=1}^{r}\left(b_{d i t 1} d_{l}(k)+b_{d i l 2} d_{l}(k-1)+\cdots\right. \\
& \left.+b_{d i h_{i}} d_{l}\left(k-n_{i}+1\right)\right)
\end{aligned}
$$

For each output, the difference equation can be transformed in a discrete state model with affine terms:

$$
\begin{aligned}
& x_{i}(k) \in \Re^{n_{i}} \\
& x_{i}(k+1)= \\
& =\left[\begin{array}{c}
a_{i 0} \cdot a_{i 1} \\
a_{i 0} \cdot a_{i 2} \\
\vdots \\
a_{i 0} \cdot a_{i n_{i}}
\end{array}\right]+\left[\begin{array}{cccc}
a_{i 1} & 1 & \cdots & 0 \\
a_{i 2} & 0 & \ddots & 0 \\
\vdots & \vdots & \ddots & 1 \\
a_{i n_{i}} & 0 & \cdots & 0
\end{array}\right] x_{i}(k) \\
& +\left[\begin{array}{cccc}
b_{i 11} & b_{i 21} & \cdots & b_{i p 1} \\
b_{i 12} & b_{i 22} & \cdots & b_{i p 2} \\
\vdots & \vdots & \ddots & \vdots \\
b_{i 1 n_{i}} & b_{i 2 n_{i}} & \cdots & b_{i p n_{i}}
\end{array}\right]\left[\begin{array}{c}
u_{1}(k) \\
u_{2}(k) \\
\vdots \\
u_{p}(k)
\end{array}\right] \\
& +\left[\begin{array}{cccc}
b_{d i 11} & b_{d i 21} & \cdots & b_{d i r 1} \\
b_{d i 12} & b_{d i 22} & \cdots & b_{d i r 2} \\
\vdots & \vdots & \ddots & \vdots \\
b_{d i 1 n_{i}} & b_{d i 2 n_{i}} & \cdots & b_{d i n_{i}}
\end{array}\right]\left[\begin{array}{c}
d_{1}(k) \\
d_{2}(k) \\
\vdots \\
d_{r}(k)
\end{array}\right] \\
& y_{i}(k)=a_{i 0}+\left[\begin{array}{llll}
1 & 0 & \cdots & 0
\end{array}\right] x_{i}(k)
\end{aligned}
$$

in matrix notation: 


$$
\begin{aligned}
x_{i}(k+1) & =a_{x i}+A_{i} x_{i}(k)+B_{i} u(k)+B_{d i} d(k) \\
y_{i}(k) & =a_{i 0}+C_{i} x_{i}(k)
\end{aligned}
$$

where $a_{x i} \in \mathfrak{R}^{\left(n_{i} \times 1\right)}, A_{i} \in \mathfrak{R}^{\left(n_{i} \times n_{i}\right)}, B_{i} \in \mathfrak{R}^{\left(n_{\mathrm{i}} \times p\right)}, B_{d i} \in$ $\Re^{\left(n_{i} \times r\right)}$ and $C_{i} \in \Re^{\left(1 \times n_{i}\right)}$.

Grouping the whole multivariable system in an unique model, we obtain:

$$
\begin{aligned}
& {\left[\begin{array}{c}
x_{1}(k+1) \\
x_{2}(k+1) \\
\vdots \\
x_{q}(k+1)
\end{array}\right]=\left[\begin{array}{c}
a_{x 1} \\
a_{x 2} \\
\vdots \\
a_{x q}
\end{array}\right]+} \\
& +\left[\begin{array}{cccc}
A_{1} & 0 & \cdots & 0 \\
0 & A_{2} & \cdots & 0 \\
\vdots & \vdots & \ddots & \vdots \\
0 & 0 & \cdots & A_{q}
\end{array}\right]\left[\begin{array}{c}
x_{1}(k) \\
x_{2}(k) \\
\vdots \\
x_{q}(k)
\end{array}\right]+ \\
& +\left[\begin{array}{c}
B_{1} \\
B_{2} \\
\vdots \\
B_{q}
\end{array}\right]\left[\begin{array}{c}
u_{1}(k) \\
u_{2}(k) \\
\vdots \\
u_{p}(k)
\end{array}\right]+\left[\begin{array}{c}
B_{d 1} \\
B_{d 2} \\
\vdots \\
B_{d q}
\end{array}\right]\left[\begin{array}{c}
d_{1}(k) \\
d_{2}(k) \\
\vdots \\
d_{r}(k)
\end{array}\right] \\
& {\left[\begin{array}{c}
y_{1}(k) \\
y_{2}(k) \\
\vdots \\
y_{q}(k)
\end{array}\right]=\left[\begin{array}{c}
a_{10} \\
a_{20} \\
\vdots \\
a_{q 0}
\end{array}\right]+} \\
& +\left[\begin{array}{cccc}
C_{1} & 0 & \cdots & 0 \\
0 & C_{2} & \cdots & 0 \\
\vdots & \vdots & \ddots & \vdots \\
0 & 0 & \cdots & C_{q}
\end{array}\right]\left[\begin{array}{c}
x_{1}(k) \\
x_{2}(k) \\
\vdots \\
x_{q}(k)
\end{array}\right]
\end{aligned}
$$

which can be represented as follows:

$$
\begin{aligned}
x(k+1) & =a_{x}+A x(k)+B u(k)+B_{d} d(k) \\
y(k) & =a_{y}+C x(k)
\end{aligned}
$$

where the $n=n_{1}+n_{2}+\cdots+n_{q}, a_{x} \in \Re^{(n \times 1)}$, $A \in \mathfrak{R}^{(n \times n)}, B \in \mathfrak{R}^{(n \times p)}, B_{d} \in \mathfrak{R}^{(n \times r)}, a_{y} \in \mathfrak{R}^{(q \times 1)}$, $C \in \Re^{(q \times n)}$ and some of the parameters $a_{i n_{i}}, b_{i 1 n_{i}}, \ldots b_{i p n_{i}}$, $b_{d i 1 n_{i}}, \ldots b_{d i r n_{i}}$ are not null. The system has been identified using input-output data, then the obtained state model will be controllable and observable.

Note that the $d$ inputs are non-manipulated ones and it cannot be used in state feedback, but that $d$ inputs and $B_{d}$ matrix can be considered in the observer formulation, thus improving the state estimation.

\subsection{State controller and observer}

In order to calculate the coefficients of the state feedback controller $K$, any state control design methodology can be applied. Discrete LQR [31,32] method is chosen, which allows optimal control weighting the dynamic response and the control action. The advantages of using discrete LQR is that this algorithm designs a controller matrix for the optimal state control. Also, discrete LQR algorithm is an easy method for the controllers design and it is possible to be applied with different state models.

In LQR method, since the target state is not null, the goal is to minimize the cost index $J$, which necessitates the tuning of state $Q$ and input $R$ weighting matrices, and the knowledge of state and input references, $x_{r}$ and $u_{r}$.

$$
\begin{aligned}
J= & \sum_{k=0}^{\infty}\left[\left(x(k)-x_{r}\right)^{T} Q\left(x(k)-x_{r}\right)+\right. \\
& \left.+\left(u(k)-u_{r}\right)^{T} R\left(u(k)-u_{r}\right)\right]
\end{aligned}
$$

However, from the point of view of real applications, the aim is to achieve a desired output value $y_{r}$, so we propose a systematic method whose objective is to obtain the state and input references, $x_{r}$ and $u_{r}$, for a desired output reference $y_{r}$.

Recalling the system state equation described in (7), the objective is to achieve $y_{r}$. Thus, the variables $x(k), u(k)$ and $y(k)$ are considered to be in steady state $\left(x_{r}, u_{r}\right.$ and $y_{r}$ ). Then it must fulfil:

$$
\begin{aligned}
& x_{r}=a_{x}+A x_{r}+B u_{r}+B_{d} d(k) \\
& y_{r}=a_{y}+C x_{r}
\end{aligned}
$$

Rearranging:

$$
\begin{aligned}
(A-I) x_{r}+B u_{r} & =-a_{x}-B_{d} d(k) \\
C x_{r} & =y_{r}-a_{y}
\end{aligned}
$$

In matrix form:

$$
\left[\begin{array}{cc}
(A-I) & B \\
C & 0
\end{array}\right]\left[\begin{array}{l}
x_{r} \\
u_{r}
\end{array}\right]=\left[\begin{array}{c}
-a_{x}-B_{d} d(k) \\
y_{r}-a_{y}
\end{array}\right]
$$

Solving for $x_{r}$ and $u_{r}$ :

$$
\left[\begin{array}{l}
x_{r} \\
u_{r}
\end{array}\right]=\left[\begin{array}{cc}
(A-I) & B \\
C & 0
\end{array}\right]^{-1}\left[\begin{array}{c}
-a_{x}-B_{d} d(k) \\
y_{r}-a_{y}
\end{array}\right]
$$

and the control action becomes:

$$
u(k)=K\left(x_{r}-x(k)\right)+u_{r}
$$

This method allows computing the state and input references, $x_{r}$ and $u_{r}$, in a systematic way. However, the method effectiveness depends on the accuracy of the estimated model, so unmodelled disturbances or modelling errors will produce steady state errors. 
On the other hand, since the state $x(k)$ is not directly accessible, a state observer is required. We choose an optimal observer [30].

Recalling the system given by (7):

$$
\begin{aligned}
x(k+1) & =a_{x}+A x(k)+B u(k)+B_{d} d(k) \\
y(k) & =a_{y}+C x(k)
\end{aligned}
$$

the observer is formulated as follows:

$$
\begin{aligned}
x_{e}(k+1)= & a_{x}+A x_{e}(k)+B u(k)+B_{d} d(k)+ \\
& +H\left(y(k)-\left(a_{y}+C x_{e}(k)\right)\right)
\end{aligned}
$$

where $H \in \Re^{(n \times q)}$.

The optimal observer [30] solves the problem of calculating a matrix $H$ which minimizes the cost index $J$ :

$$
J(H)=\alpha^{T}(A-H C)(A-H C)^{T} \alpha
$$

$\forall \alpha \in \Re^{n}$

It is verified in [30] that for any value of $\alpha$, it fulfills:

$$
H=A C^{T}\left(C C^{T}\right)^{-1}
$$

As the matrix $C$ is defined in this particular state representation, it holds that:

$$
C C^{T}=I
$$

so that:

$$
H=A C^{T}
$$

The separation principle holds between optimal observer and control design.

\subsection{Incremental state model}

As commented before, the proposed method to obtain the state and inputs references, $x_{r}$ and $u_{r}(11)$, can produce steady state errors in presence of modelling errors. This problem can be solved by incremental state model [30].

Applying the discrete state model described in (7), at the previous sample $(k-1)$, we get:

$$
\begin{aligned}
& x(k)=a_{x}+A x(k-1)+B u(k-1)+B_{d} d(k-1) \\
& y(k-1)=a_{y}+C x(k-1)
\end{aligned}
$$

Subtracting (18) from (7), we get:

$$
\begin{aligned}
& x(k+1)-x(k)=A(x(k)-x(k-1))+ \\
& +B(u(k)-u(k-1))+B_{d}(d(k)-d(k-1)) \\
& y(k)-y(k-1)=C(x(k)-x(k-1))
\end{aligned}
$$

where the affine terms are cancelled. Defining the incremental state $\Delta x$, the incremental manipulated input $\Delta u$ and the incremental non-manipulated measurable input $\Delta d$ as follows:

$$
\begin{aligned}
& \Delta x(k)=x(k)-x(k-1) \\
& \Delta u(k)=u(k)-u(k-1) \\
& \Delta d(k)=d(k)-d(k-1)
\end{aligned}
$$

Substituting (20) into (19), we obtain:

$$
\begin{aligned}
\Delta x(k+1) & =A \Delta x(k)+B \Delta u(k)+B_{d} \Delta d(k) \\
y(k) & =y(k-1)+C \Delta x(k)
\end{aligned}
$$

New $p$ states are introduced to complete the formulation, verifying that:

$$
\begin{aligned}
& y(k+1)=y(k)+C \Delta x(k+1)= \\
& =y(k)+C\left(A \Delta x(k)+B \Delta u(k)+B_{d} \Delta d(k)\right)
\end{aligned}
$$

We define a new expanded incremental state vector $x_{a} \in$ $\mathfrak{R}^{(q+n)}$, obtaining the new state model as follows:

$$
x_{a}(k)=\left[\begin{array}{c}
y(k) \\
\Delta x(k)
\end{array}\right]
$$

$$
\begin{aligned}
& {\left[\begin{array}{c}
y(k+1) \\
\Delta x(k+1)
\end{array}\right]=\left[\begin{array}{cc}
I & C A \\
0 & A
\end{array}\right]\left[\begin{array}{c}
y(k) \\
\Delta x(k)
\end{array}\right]+} \\
& +\left[\begin{array}{c}
C B \\
B
\end{array}\right] \Delta u(k)+\left[\begin{array}{c}
C B_{d} \\
B_{d}
\end{array}\right] \Delta d(k) \\
& y(k)=\left[\begin{array}{ll}
I & 0
\end{array}\right]\left[\begin{array}{c}
y(k) \\
\Delta x(k)
\end{array}\right]
\end{aligned}
$$

In matrix notation, the expanded state model becomes:

$$
\begin{aligned}
x_{a}(k+1) & =A_{a} x_{a}(k)+B_{a} \Delta u(k)+B_{d a} \Delta d(k) \\
y(k) & =C_{a} x_{a}(k)
\end{aligned}
$$

\subsection{Proposed incremental state controller and observer}

Incremental state feedback controllers can be designed by any method. We propose discrete LQR method [31,32] due to its advantages described in Section 3.2. In addition, we use the same control method in both state models to show the advantages of the proposed incremental state model with respect to the non incremental one. 
If the control objective is to approach a steady state output $y_{r}$, this is equivalent to achieve the reference expanded state in incremental model [30]:

$$
x_{a r}=\left[\begin{array}{c}
y_{r} \\
\Delta x_{r}
\end{array}\right]=\left[\begin{array}{c}
y_{r} \\
0
\end{array}\right]
$$

It should be noted that the reference expanded state comes naturally without the necessity of performing any calculations. Then the control action is described as:

$$
\begin{aligned}
& \Delta u(k)=K\left(x_{a r}-x_{a}(k)\right)= \\
& =\left[\begin{array}{ll}
K_{y} & K_{x}
\end{array}\right]\left(\left[\begin{array}{c}
y_{r} \\
0
\end{array}\right]-\left[\begin{array}{c}
y(k) \\
\Delta x(k)
\end{array}\right]\right)= \\
& =\left[\begin{array}{ll}
K_{y} & K_{x}
\end{array}\right]\left[\begin{array}{c}
y_{r}-y(k) \\
-\Delta x(k)
\end{array}\right] \\
& u(k)=u(k-1)+\Delta u(k) \\
& K \in \Re^{(p \times(q+n))}, K_{y} \in \Re^{(p \times q)}, K_{x} \in \Re^{(p \times n)}
\end{aligned}
$$

If the feedback system is stable and the steady state is approached, the following conditions are fulfilled:

$$
\lim _{k \rightarrow \infty} \Delta u(k)=0, \lim _{k \rightarrow \infty} \Delta x(k)=0
$$

and thus

$$
\lim _{k \rightarrow \infty} K_{y}\left(y_{r}-y(k)\right)=0
$$

So, if $K_{y}$ is of rank $q$, it verifies that:

$$
\lim _{k \rightarrow \infty} y(k)=y_{r}
$$

therefore, the controlled system has zero steady state error.

Note that control action (26) is calculated in incremental form (25), which is equivalent to apply an integral control action.

On the other hand, we suppose that the output $y(k)$ is measurable, but the incremental state $\Delta x(k)$ is not directly accessible, then a state observer is required.

The estimated incremental state can be obtained by the state observer described in previous Section 3.2:

$$
\Delta x_{e}(k)=x_{e}(k)-x_{e}(k-1)
$$

or directly by the incremental state observer [30], which is formulated as follows:

$$
\begin{aligned}
& \Delta x_{e}(k+1)=A \Delta x_{e}(k)+B \Delta u(k)+ \\
& +B_{d} \Delta d(k)+H\left(y(k)-y(k-1)-C \Delta x_{e}(k)\right)
\end{aligned}
$$

where $H \in \Re^{(n \times q)}$ coefficients are obtained by optimal state observer design [30], as described in (17). follows:

Finally, the expanded estimated state is obtained as

$$
x_{a e}(k)=\left[\begin{array}{c}
y(k) \\
\Delta x_{e}(k)
\end{array}\right]
$$

\section{PROPOSED CONTROLLERS AND RESULTS ANALYSIS}

In this section, we apply the proposed linear control methods to the nonlinear wind turbine model described in Section II, comparing the performances of the incremental state model control with those of the non incremental one, in order to choose the most adequate solution.

That wind turbine model works in continuous time, but the controller has been developed in discrete time, so a sampler and zero order holding device have been added to the model. All system variables are supposed to be ideally measured.

In order to obtain the proposed linear controllers, we have identified, by the least squares method, the following linearized system of the nonlinear wind turbine model, around the operating point defined in Table III, supposing the proposed sampling time is $T=0.05 \mathrm{~s}$ :

$$
\begin{aligned}
\omega_{r}(k+1)= & 0.0012+2.7318 \omega_{r}(k)- \\
& -2.4152 \omega_{r}(k-1)+0.6228 \omega_{r}(k-2)+ \\
& +0.0604 \omega_{r}(k-3)+0.4094 v_{w}(k) \\
& -0.7407 v_{w}(k-1)+0.3054 v_{w}(k-2)+ \\
& +0.0268 v_{w}(k-3)-0.00005 \beta_{r}(k)- \\
& -0.0002 \beta_{r}(k-1)-0.0001 \beta_{r}(k-2)- \\
& -0.000007 \beta_{r}(k-3)-0.0363 I_{f_{r}}(k)- \\
& -0.0013 I_{f_{r}}(k-1)+0.0336 I_{f_{r}}(k-2)+ \\
& +0.0025 I_{f_{r}}(k-3)
\end{aligned}
$$

$$
\begin{aligned}
P_{g}(k+1)= & -0.7204+2.1484 P_{g}(k)- \\
& -0.7796 P_{g}(k-1)-0.9047 P_{g}(k-2)+ \\
& +0.5357 P_{g}(k-3)+13.0373 v_{w}(k)- \\
& -15.9819 v_{w}(k-1)-4.5794 v_{w}(k-2)+ \\
& +7.5706 v_{w}(k-3)-0.0014 \beta_{r}(k)- \\
& -0.0065 \beta_{r}(k-1)-0.0051 \beta_{r}(k-2)- \\
& -0.0011 \beta_{r}(k-3)+169.3539 I_{f_{r}}(k)- \\
& -212.753 I_{f_{r}}(k-1)-57.8343 I_{f_{r}}(k-2)+ \\
& +101.6462 I_{f_{r}}(k-3)
\end{aligned}
$$




\subsection{Discrete state model}

From the input/output identified model (equations 30 and 31) and following the methodology described in previous Section 3.1, the wind turbine discrete state model (7) is obtained:

$$
\begin{aligned}
x(k+1) & =a_{x}+A x(k)+B u(k)+B_{d} d(k) \\
y(k) & =a_{y}+C x(k)
\end{aligned}
$$

where:

$$
\begin{aligned}
& x(k) \in \mathfrak{R}^{8} \\
& y(k)=\left[\begin{array}{c}
\omega_{r}(k) \\
P_{g}(k)
\end{array}\right], u(k)=\left[\begin{array}{c}
\beta_{r}(k) \\
I_{f_{r}}(k)
\end{array}\right], d(k)=v_{w}(k) \\
& a_{x}=\left[\begin{array}{c}
0.0034 \\
-0.0030 \\
0.0008 \\
0.0001 \\
-1.5478 \\
0.5617 \\
0.6518 \\
-0.3859
\end{array}\right], B_{d}=\left[\begin{array}{c}
0.4094 \\
-0.7407 \\
0.3054 \\
0.0268 \\
13.0373 \\
-15.9819 \\
-4.5794 \\
7.5706
\end{array}\right] \\
& B=\left[\begin{array}{cc}
-0.00005 & -0.0363 \\
-0.0002 & -0.0013 \\
-0.0001 & 0.0336 \\
-0.000007 & 0.0025 \\
-0.0014 & 169.3539 \\
-0.0065 & -212.7530 \\
-0.0051 & -57.8343 \\
-0.0011 & 101.6462
\end{array}\right] \\
& A=\left[\begin{array}{cccccccc}
2.7318 & 1 & 0 & 0 & 0 & 0 & 0 & 0 \\
-2.4152 & 0 & 1 & 0 & 0 & 0 & 0 & 0 \\
0.6228 & 0 & 0 & 1 & 0 & 0 & 0 & 0 \\
0.0604 & 0 & 0 & 0 & 0 & 0 & 0 & 0 \\
0 & 0 & 0 & 0 & 2.1484 & 1 & 0 & 0 \\
0 & 0 & 0 & 0 & -0.7796 & 0 & 1 & 0 \\
0 & 0 & 0 & 0 & -0.9047 & 0 & 0 & 1 \\
0 & 0 & 0 & 0 & 0.5357 & 0 & 0 & 0
\end{array}\right] \\
& a_{y}=\left[\begin{array}{c}
0.0012 \\
-0.7204
\end{array}\right] \\
& C=\left[\begin{array}{llllllll}
1 & 0 & 0 & 0 & 0 & 0 & 0 & 0 \\
0 & 0 & 0 & 0 & 1 & 0 & 0 & 0
\end{array}\right]
\end{aligned}
$$

Note that wind speed $v_{w}$ is a non-manipulated input. As described in Section 3.1, it cannot be used in state feedback, but $v_{w}$ input and $B_{d}$ matrix can be considered in the observer.

The controller algorithm is designed by the discrete LQR method, using the following positive definite weighting matrices:

$$
\begin{aligned}
& Q=\operatorname{diag}\left(\left[\begin{array}{llllllll}
1 & 1 & 1 & 1 & 1 & 1 & 1 & 1
\end{array}\right]\right) \\
& R=\operatorname{diag}\left(\left[\begin{array}{ll}
1 & 1
\end{array}\right]\right)
\end{aligned}
$$

We obtain the state and input references as described in equation (11):

$$
\left[\begin{array}{l}
x_{r} \\
u_{r}
\end{array}\right]=\left[\begin{array}{cc}
(A-I) & B \\
C & 0
\end{array}\right]^{-1}\left[\begin{array}{c}
-a_{x}-B_{d} d(k) \\
y_{r}-a_{y}
\end{array}\right]
$$

As described in equation (12), the control action becomes:

$$
u(k)=K\left(x_{r}-x(k)\right)+u_{r}
$$

with:

$$
\begin{aligned}
& K=\left[\begin{array}{cccc}
-79.5628 & -77.8970 & -76.0858 & -74.136 \\
-0.0002 & -0.0001 & -0.00004 & 0.00005
\end{array}\right. \\
& \left.\begin{array}{cccc}
-2.9806 & -2.9068 & -2.8207 & -2.7252 \\
0.0073 & 0.0020 & -0.0024 & -0.0006
\end{array}\right]
\end{aligned}
$$

And the state observer, defined in equation (13) is:

$$
\begin{aligned}
x_{e}(k+1)= & a_{x}+A x_{e}(k)+B u(k)+B_{d} d(k)+ \\
& +H\left(y(k)-a_{y}-C x_{e}(k)\right)
\end{aligned}
$$

with:

$$
H=\left[\begin{array}{cc}
2.7318 & 0 \\
-2.4152 & 0 \\
0.6228 & 0 \\
0.0604 & 0 \\
0 & 2.1484 \\
0 & -0.7796 \\
0 & -0.9047 \\
0 & 0.5357
\end{array}\right]
$$

\subsection{Incremental state model}

Wind turbine incremental state model equations are described by equation (24):

$$
\begin{aligned}
x_{a}(k+1) & =A_{a} x_{a}(k)+B_{a} \Delta u(k)+B_{d a} \Delta d(k) \\
y(k) & =C_{a} x_{a}(k)
\end{aligned}
$$

where:

$$
\begin{aligned}
& x_{a}(k)=\left[\begin{array}{c}
y(k) \\
\Delta x(k)
\end{array}\right], y(k)=\left[\begin{array}{c}
\omega_{r}(k) \\
P_{g}(k)
\end{array}\right], \\
& u(k)=\left[\begin{array}{c}
\beta_{r}(k) \\
I_{f_{r}}(k)
\end{array}\right], d(k)=v_{w}(k) \\
& \Delta x(k) \in \mathfrak{R}^{8}, A_{a} \in \mathfrak{R}^{10 \times 10}, \\
& B_{a} \in \mathfrak{R}^{10 \times 2}, B_{d a} \in \mathfrak{R}^{10 \times 1}, C_{a} \in \mathfrak{R}^{2 \times 10}
\end{aligned}
$$


The controller algorithm is designed by the discrete LQR method, using the following positive definite weighting matrices:

$$
\begin{aligned}
& Q=\operatorname{diag}\left(\left[\begin{array}{llllllllll}
1 & 1 & 1 & 1 & 1 & 1 & 1 & 1 & 1 & 1
\end{array}\right]\right) \\
& R=\operatorname{diag}\left(\left[\begin{array}{lll}
1 & 1
\end{array}\right]\right)
\end{aligned}
$$

As described in equations (25) and (26), the control action is:

$$
\begin{aligned}
& \Delta u(k)=K\left(x_{a r}-x_{a}(k)\right) \\
& u(k)=u(k-1)+\Delta u(k)
\end{aligned}
$$

with:

$$
\begin{aligned}
& K=\left[\begin{array}{cccc}
-0.9048 & -0.0209 & -721.0133 & -666.949 \\
-0.0001 & 0.0025 & -0.0636 & -0.0590
\end{array}\right. \\
& \ldots \begin{array}{ccc}
-613.4391 & -561.5192 & -0.7790 \\
-0.0545 & -0.0501 & 0.0141
\end{array} \\
& \left.\ldots \begin{array}{ccc}
-0.5897 & -0.3961 & -0.2050 \\
0.0075 & 0.0018 & 0.0020
\end{array}\right]
\end{aligned}
$$

And the state observer, described in equations (28) and (29), is:

$$
\begin{aligned}
\Delta x_{e}(k+1)= & A \Delta x_{e}(k)+B \Delta u(k)+B_{d} \Delta d(k)+ \\
& +H\left(y(k)-y(k-1)-C \Delta x_{e}(k)\right) \\
x_{a e}(k)= & {\left[\begin{array}{c}
y(k) \\
\Delta x_{e}(k)
\end{array}\right] }
\end{aligned}
$$

with the same $H$ matrix obtained in previous Section 4.1.

\subsection{Results and analysis}

In all the figures presented in this section, the incremental state model response is shown in continuous line and the non incremental state model response in dotted line. If reference signal is represented, it is shown in dashed line.

Table IV shows the eigenvalues of the system with and without the proposed controllers. It can be observed that the eigenvalues of the system without control, the system controlled by discrete LQR and the system controlled by incremental LQR are very similar. This means that the controlled systems have similar dynamics. However, the objective of the proposed controllers is not modifying the dynamic behaviour of the wind turbine, which is considered adequate, but to guarantee the disturbances rejection.

Fig. 4 shows the dynamic behaviour of the wind turbine model, controlled by discrete and incremental LQR algorithms, subjected to changes in the reference signal of rotor angular speed $\omega_{r}$ and generated power $P_{g}$.
Table IV. Eigenvalues of the system with and without the proposed controllers.

\begin{tabular}{lcc}
\hline $\begin{array}{l}\text { Eigenvalues } \\
\text { of the system } \\
\text { without control }\end{array}$ & $\begin{array}{c}\text { Eigenvalues } \\
\text { of the system } \\
\text { with discrete } \\
\text { LQR control }\end{array}$ & $\begin{array}{c}\text { Eigenvalues } \\
\text { of the system } \\
\text { with incremetal } \\
\text { LQR control }\end{array}$ \\
\hline 0.9784 & 0.9780 & 0.9774 \\
0.9772 & $0.9304+0.0567 \mathrm{i}$ & $0.9335+0.1003 \mathrm{i}$ \\
0.9230 & $0.9304-0.0567 \mathrm{i}$ & $0.9335-0.1003 \mathrm{i}$ \\
0.9221 & 0.9224 & 0.9226 \\
0.9052 & 0.8940 & $0.8766+0.0411 \mathrm{i}$ \\
0.9046 & 0.0000 & $0.8766-0.0411 \mathrm{i}$ \\
-0.0739 & -0.0739 & 0.5685 \\
-0.6565 & -0.6565 & 0.0000 \\
& & -0.0739 \\
& & -0.6565 \\
\hline
\end{tabular}
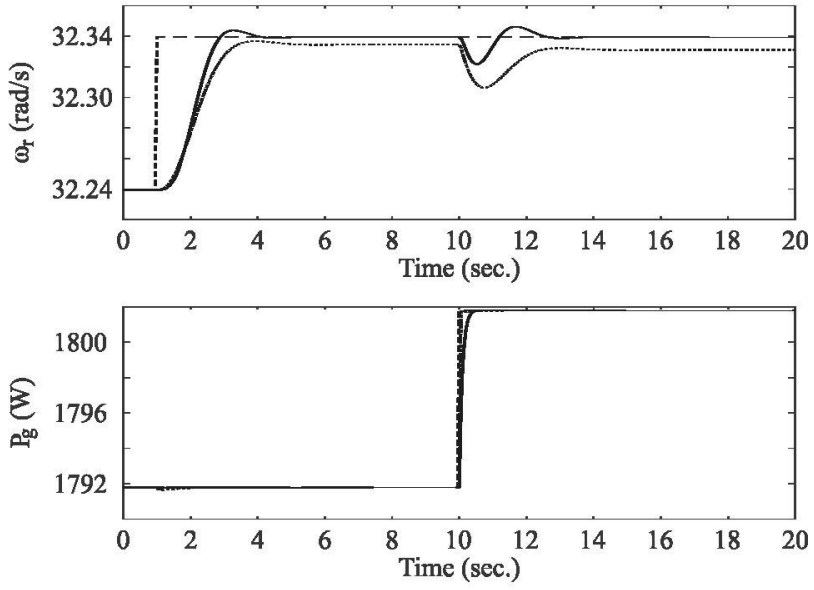

Fig. 4. Rotor angular speed $\omega_{r}$ and generated power $P_{g}$ responses to changes in references signals.

It can be observed that, both controlled responses present similar dynamic behaviour. However, even with the adjustment method of state and input references $x_{r}$ and $u_{r}$, the controlled system by discrete LQR presents small steady state errors, because of modelling errors, which do not appear in the controlled system by incremental state model.

In order to compare the performances of the controllers in presence of disturbances, changes in wind speed $v_{w}$ and line resistance $R_{l}$ are applied. Fig. 5 shows the dynamic behaviour of the wind turbine model, controlled by discrete and incremental LQR algorithms, subjected to changes in the reference signal of rotor angular speed $\omega_{r}$ and generated power $P_{g}$. Moreover, it is subjected to step disturbances in wind speed from $v_{w}=$ $10.55 \mathrm{~m} / \mathrm{s}$ to $v_{w}=10.50 \mathrm{~m} / \mathrm{s}$ at $t=10 \mathrm{~s}$ and line resistance 

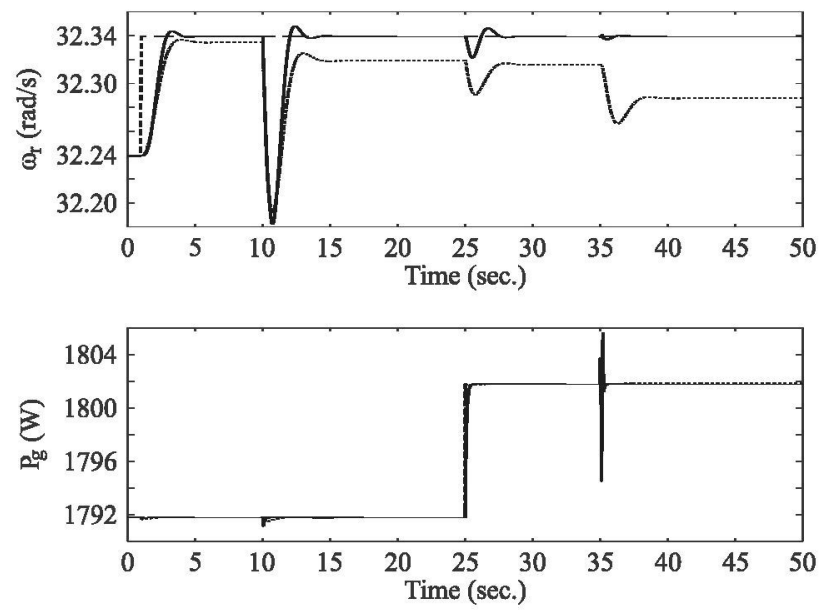

Fig. 5. Rotor angular speed $\omega_{r}$ and generated power $P_{g}$ responses to changes in references signals and disturbances.
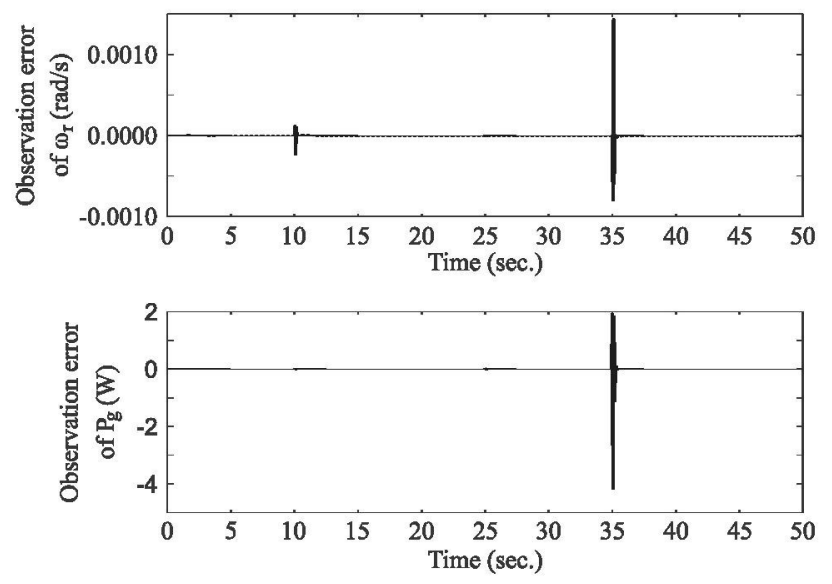

Fig. 6. Rotor angular speed $\omega_{r}$ and generated power $P_{g}$ observation errors

from $R_{l}=4.00 \Omega$ to $R_{l}=4.01 \Omega$ at $t=35 \mathrm{~s}$. Fig. 6 shows the Observation Error (OE).

It can be observed that, even both controllers produces similar dynamic behaviour, the controlled system by discrete $L Q R$ presents steady state errors. On the other hand, incremental state feedback method produces zero steady state error even in presence of modelling errors, nonlinearities and unmodelled disturbances.

It can be checked that the optimal state observer produces fast convergence to zero observation error and, because of considering $v_{w}$ input and $B_{d}$ matrix in the state observer formulation, the observation error produced by wind speed $v_{w}$ disturbance in $t=10 \mathrm{~s}$ has low amplitude. The observation error produced with the non incremental state model presents a small steady state error making impossible the complete convergence
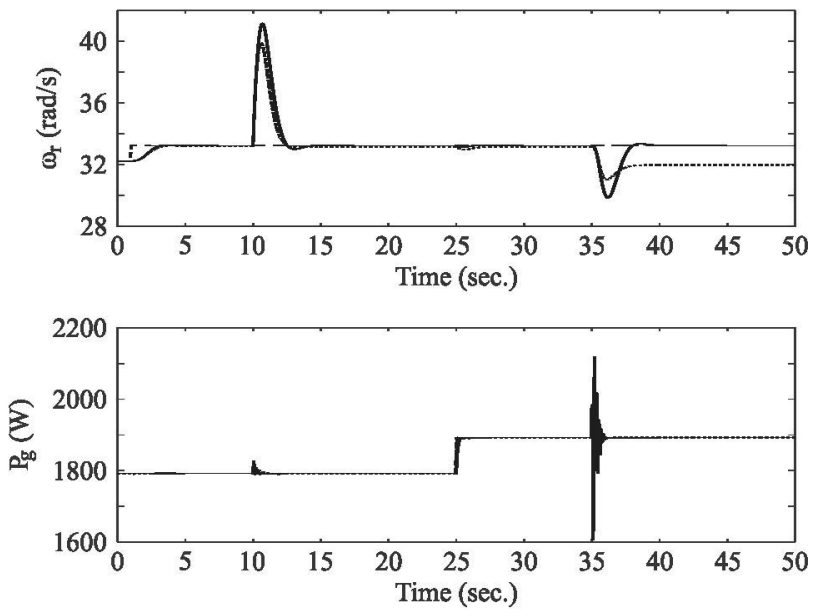

Fig. 7. Rotor angular speed $\omega_{r}$ and generated power $P_{g}$ responses to changes in references signals and excessive disturbances.

between the estimated state and the real one. Incremental state model presents zero steady state error also in the observation error.

In order to compare the performances of the controllers in the above rated zone and in presence of large disturbances, excessive changes in wind speed $v_{w}$ and line resistance $R_{l}$ are applied. Fig. 7 shows the dynamic behaviour of the wind turbine model, controlled by discrete and incremental LQR algorithms, subjected to changes in the reference signal of rotor angular speed $\omega_{r}$ and generated power $P_{g}$. Moreover, it is subjected to step disturbances in wind speed from $v_{w}=10.55 \mathrm{~m} / \mathrm{s}$ to $v_{w}=13.00 \mathrm{~m} / \mathrm{s}$ at $t=10 \mathrm{~s}$ and line resistance from $R_{l}=4.00 \Omega$ to $R_{l}=4.50 \Omega$ at $t=35 \mathrm{~s}$.

It can be seen that both state models control algorithms can provide stability in the full range of the above rated zone, but the nonlinearities of the model have changed the dynamic behaviour of the controlled system. Also, the steady state error of the controlled system by discrete $\mathrm{LQR}$ is too high for a good nominal operation, so zero steady state error must be obtained and it has been got by the incremental state model control.

\section{CONCLUSIONS}

In this paper, we have proposed a multivariable optimal control of wind turbines using a new methodology based on incremental state model, with a LQR control and an optimal observer.

The incremental model offers several advantages to solve traditional state models problems. 
- First, control action in incremental state model is equivalent to introduce an integral action, cancelling the steady state errors produced by the non incremental formulation.

- Second, in the classical LQR control, the reference state should be calculated, while in the incremental LQR control, the reference state is directly the output reference with a null reference for the state increase.

The application of the proposed linear controller to the simulated wind turbine nonlinear model has shown that incremental LQR control presents a good transient response and zero steady state error, even in presence of modelling errors, nonlinearities and disturbances.

We propose as future work, to design an extension of the proposed control method using a non-linear approach by the T-S fuzzy model. Wind turbines are non-linear systems with strong nonlinearities in the slow wind zone, thus a non-linear approach of the incremental state control method based in T-S fuzzy models could obtain better results in the full operation range of the wind turbine, with the same advantages of the proposed linear controller.

\section{APPENDIX}

\subsection{Nomenclature and list of abbreviations}

Table A1. Nomenclature.

$\beta_{r} \quad$ Blades angle reference

$I_{f_{r}} \quad$ Field current reference

$v_{w} \quad$ Wind speed

$R_{L} \quad$ Electrical line resistance

$\omega_{r} \quad$ Rotor angular speed

$P_{g} \quad$ Generated power

$\beta \quad$ Blades angle

$I_{f} \quad$ Field current

$C_{p} \quad$ Power coefficient

$C_{q} \quad$ Mechanical coefficient

$p \quad$ Number of system manipulated inputs

$u \quad$ System manipulated inputs

$r \quad$ Number of system measu rable distu rbances

$d \quad$ System measurable disturbances

$q \quad$ Number of system outputs

$y$ System outputs

$x \quad$ System states

$n \quad$ Number of system states

$a_{x} \quad$ State affine terms matrix

$A \quad$ State dynamic matrix

$B$ Input dynamic matrix

$B_{d} \quad$ Disturbance dynamic matrix

$a_{y} \quad$ Output affine terms matrix

C Output matrix

$K \quad$ State controller matrix

$J$ Cost index function

$Q \quad$ State weighting matrix

$R \quad$ Input weighting matrix

$x_{r} \quad$ State reference

$u_{r} \quad$ Input reference

$y_{r} \quad$ Output reference

$H$ State observer matrix

$T \quad$ Sampling time

$t$ Time

Table A2. List of abbreviations.

LQR Linear Quadratic Regulator

PI Proportional Integral

PID Proportional Integral Derivative

QFT Quantitative Feedback Theory

CART 3 Controls Advanced Research Turbine 3-bladed

MSISO multiple single-input single-output

MIMO multiple-input multiple-output

DEMM Difference Equation Matrix Model

DMC Dynamic Matrix Control

RGPC Recursive Generalized Predictive Control

OE Observation Error

T-S Takagi-Sugeno 